\title{
A NOTE ON THE ASYMPTOTIC BEHAVIOR OF RADIAL SOLUTIONS TO QUASILINEAR ELLIPTIC EQUATIONS WITH A HARDY POTENTIAL
}

\author{
KENTA ITAKURA AND SATOSHI TANAKA \\ (Communicated by Wenxian Shen)
}

Abstract. The quasilinear elliptic equation with a Hardy potential

$$
\operatorname{div}\left(|x|^{\alpha}|\nabla u|^{p-2} \nabla u\right)+\frac{\mu}{|x|^{p-\alpha}}|u|^{p-2} u=0 \quad \text { in } \mathbf{R}^{N}-\{0\}
$$

is considered, where $N \in \mathbf{N}, p>1$ and $\alpha \in \mathbf{R}, \mu \in \mathbf{R}-\{0\}$. In this note, the asymptotic behaviors of radial solutions are obtained divided into three case $\mu<|(N-p+\alpha) / p|^{p}, \mu=|(N-p+\alpha) / p|^{p}$ and $\mu>|(N-p+\alpha) / p|^{p}$. This equation also appears as the Euler-Lagrange equation related to the weighted Hardy inequality

$$
\int_{\Omega}|\nabla u(x)|^{p}|x|^{\alpha} d x \geq\left|\frac{N-p+\alpha}{p}\right|^{p} \int_{\Omega}|u(x)|^{p}|x|^{\alpha-p} d x
$$

for $u \in C_{c}^{\infty}\left(\mathbf{R}^{N}\right)$ and $N-p+\alpha \neq 0$, where $\Omega$ is a domain of $\mathbf{R}^{N}$.

The rectifiability of oscillatory solutions to the ordinary differential equation with one-dimensional $p$-Laplacian is also studied, and an answer to an open problem is given.

\section{INTRODUCTION}

We consider the quasilinear elliptic equation with a Hardy potential

$$
\operatorname{div}\left(|x|^{\alpha}|\nabla u|^{p-2} \nabla u\right)+\frac{\mu}{|x|^{p-\alpha}}|u|^{p-2} u=0 \quad \text { in } \mathbf{R}^{N}-\{0\},
$$

where $N \in \mathbf{N}, p>1$ and $\alpha \in \mathbf{R}, \mu \in \mathbf{R}-\{0\}$. Radial solutions of (1.1) satisfy

$$
\left(\phi_{p}\left(u^{\prime}\right)\right)^{\prime}+\frac{N-1+\alpha}{r} \phi_{p}\left(u^{\prime}\right)+\frac{\mu}{r^{p}} \phi_{p}(u)=0, \quad r>0,
$$

where $\phi_{p}(t):=|t|^{p-2} t$ for $t \in \mathbf{R}$. When $p=2$, equation (1.1) is reduced to the linear equation

$$
\operatorname{div}\left(|x|^{\alpha} \nabla u\right)+\frac{\mu}{|x|^{2-\alpha}} u=0, \quad \text { in } \mathbf{R}^{N}-\{0\},
$$

and we know two fundamental solutions of (1.3), because the radial version of (1.3) is an Euler differential equation

$$
u^{\prime \prime}+\frac{N-1+\alpha}{r} u^{\prime}+\frac{\mu}{r^{2}} u=0, \quad r>0 .
$$

Received by the editors February 23, 2021.

2020 Mathematics Subject Classification. Primary 35J92, 35B40, 34C10.

Key words and phrases. Quasilinear, elliptic equation, radial solutions, asymptotic behavior, Hardy potential, $p$-Laplacian.

The second author was supported by JSPS KAKENHI Grant Number 19K03595 and 17H01095. 
The characteristic equation of (1.4)

$$
\lambda^{2}+(N-2+\alpha) \lambda+\mu=0
$$

has two roots

$$
\lambda=\frac{-(N-2+\alpha) \pm \sqrt{(N-2+\alpha)^{2}-4 \mu}}{2} .
$$

Then we have exact solutions of (1.4) as follows:

(i) if $\mu>(N-2+\alpha)^{2} / 4$, then

$$
\begin{aligned}
u(r)=C_{1} r^{-\frac{N-2+\alpha}{2}} \sin \left(\frac{\sqrt{4 \mu-(N-2+\alpha)^{2}}}{2} \log r\right) \\
\quad+C_{2} r^{-\frac{N-2+\alpha}{2}} \cos \left(\frac{\sqrt{4 \mu-(N-2+\alpha)^{2}}}{2} \log r\right)
\end{aligned}
$$

(ii) if $\mu=(N-2+\alpha)^{2} / 4$, then

$$
u(r)=C_{1} t^{-\frac{N-2+\alpha}{2}}+C_{2} t^{-\frac{N-2+\alpha}{2}} \log r ;
$$

(iii) if $\mu<(N-2+\alpha)^{2} / 4$, then

$$
u(r)=C_{1} r \frac{-(N-2+\alpha)-\sqrt{(N-2+\alpha)^{2}-4 \mu}}{2}+C_{2} r \frac{-(N-2+\alpha)+\sqrt{(N-2+\alpha)^{2}-4 \mu}}{2} .
$$

Here, $C_{1}$ and $C_{2}$ are arbitrary constants.

On the other hand, all solutions of (1.2) cannot be expressed as exact solutions. In this note, we give the asymptotic behavior of solutions to (1.2). First we note that the global existence and uniqueness result holds for equation (1.2), which will be shown in Section 2.

Proposition 1.1. For each $u_{0}, u_{1} \in \mathbf{R}$ and $r_{0}>0$, the initial value problem (1.2) with

$$
u\left(r_{0}\right)=u_{0}, \quad u^{\prime}\left(r_{0}\right)=u_{1}
$$

has a unique solution on $(0, \infty)$.

The main results in this note are as follows.

Theorem 1.1. Assume that $\mu>|(N-p+\alpha) / p|^{p}$. Then, for each nontrivial solution $u(r)$ of (1.2), there exist sign-changing periodic functions $C, S$ with some period $L>0$ such that

$$
u(r)=r^{-\frac{N-p+\alpha}{p}} C(\log r), \quad u^{\prime}(r)=r^{-\frac{N-p+\alpha}{p}-1} S(\log r), \quad r>0 .
$$

Theorem 1.2. Assume that $\mu=|(N-p+\alpha) / p|^{p}$. Let $u(r)$ be a nontrivial solution of (1.2) and let $r_{0}>0$. Then the following (i) and (ii) hold:

(i) If $r_{0} u^{\prime}\left(r_{0}\right)=-((N-p+\alpha) / p) u\left(r_{0}\right)$, then

$$
u(r)=u\left(r_{0}\right) r_{0}^{\frac{N-p+\alpha}{p}} r^{-\frac{N-p+\alpha}{p}}, \quad r>0 ;
$$

(ii) If $r_{0} u^{\prime}\left(r_{0}\right) \neq-((N-p+\alpha) / p) u\left(r_{0}\right)$, then there exist constants $c_{1} \neq 0$ and $c_{2} \neq 0$ such that

$$
\lim _{r \rightarrow \infty} \frac{u(r)}{(\log r)^{\frac{2}{p}} r^{-\frac{N-p+\alpha}{p}}}=c_{1}, \quad \lim _{r \rightarrow \infty} \frac{u^{\prime}(r)}{(\log r)^{\frac{2}{p}}\left(r^{-\frac{N-p+\alpha}{p}}\right)^{\prime}}=c_{1}
$$


and

$$
\lim _{r \rightarrow 0^{+}} \frac{u(r)}{(-\log r)^{\frac{2}{p}} r^{-\frac{N-p+\alpha}{p}}}=c_{2}, \quad \lim _{r \rightarrow 0^{+}} \frac{u^{\prime}(r)}{(-\log r)^{\frac{2}{p}}\left(r^{-\frac{N-p+\alpha}{p}}\right)^{\prime}}=c_{2} .
$$

Theorem 1.3. Assume that $\mu<|(N-p+\alpha) / p|^{p}$. Let $u(r)$ be a nontrivial solution of (1.2) and let $r_{0}>0$. Then the equation

$$
(p-1)|\lambda|^{p}+(N-p+\alpha)|\lambda|^{p-2} \lambda+\mu=0
$$

has exactly two real roots $\lambda_{1}, \lambda_{2}$ with $\lambda_{1}<\lambda_{2}$ and the following (i) and (ii) hold:

(i) If $r_{0} u^{\prime}\left(r_{0}\right)=\lambda_{i} u\left(r_{0}\right)$ for some $i \in\{1,2\}$, then

$$
u(r)=u\left(r_{0}\right) r_{0}^{-\lambda_{i}} r^{\lambda_{i}}, \quad r>0 ;
$$

(ii) If $r_{0} u^{\prime}\left(r_{0}\right) \neq-((N-p+\alpha) / p) u\left(r_{0}\right)$ for each $i \in\{1,2\}$, then there exist constants $c_{1} \neq 0$ and $c_{2} \neq 0$ such that

$$
\lim _{r \rightarrow \infty} \frac{u(r)}{r^{\lambda_{2}}}=c_{1}, \quad \lim _{r \rightarrow \infty} \frac{u^{\prime}(r)}{\left(r^{\lambda_{2}}\right)^{\prime}}=c_{1}
$$

and

$$
\lim _{r \rightarrow 0^{+}} \frac{u(r)}{r^{\lambda_{1}}}=c_{2}, \quad \lim _{r \rightarrow 0^{+}} \frac{u^{\prime}(r)}{\left(r^{\lambda_{1}}\right)^{\prime}}=c_{2} .
$$

Moreover, the following (a)-(c) hold:

(a) if $\mu<0$, then $\lambda_{1}<0<\lambda_{2}$;

(b) if $\mu>0$ and $N-p+\alpha>0$, then $\lambda_{1}<\lambda_{2}<0$;

(c) if $\mu>0$ and $N-p+\alpha<0$, then $0<\lambda_{1}<\lambda_{2}$.

Elliptic partial differential equations involving the operator

$$
\Delta_{p} u+\frac{\mu}{|x|^{p}}|u|^{p-2} u
$$

have been studied by many authors, where $\Delta_{p} u=\operatorname{div}\left(|\nabla u|^{p-2} \nabla u\right)$ is the $p$ Laplacian. See, for example, [2, 5, 7, 9, 11, 13.

The number $|(N-p+\alpha) / p|^{p}$ appearing in Theorems 1.1 1.3 is the best constant of the weighted Hardy inequality

$$
\int_{\Omega}|\nabla u(x)|^{p}|x|^{\alpha} d x \geq\left|\frac{N-p+\alpha}{p}\right|^{p} \int_{\Omega}|u(x)|^{p}|x|^{\alpha-p} d x
$$

for $u \in C_{c}^{\infty}\left(\mathbf{R}^{N}\right)$ and $N-p+\alpha \neq 0$, where $\Omega$ is a domain of $\mathbf{R}^{N}$. See, for example, Abdellaoui, Colorado and Peral [1] and Horiuchi and Kumlin [8]. Equation (1.1) with $\mu=|(N-p+\alpha) / p|^{p}$ is the corresponding Euler-Lagrange equation for (1.12) and $|x|^{-\frac{N-p+\alpha}{p}}$ is a solution. (This solution is also obtained in Theorem 1.2 (i).)

When $p=2$, equation (1.9) is reduced to (1.5). The number $((N-2+\alpha) / 2)^{2}-\mu$ is the discriminant of (1.5), that is, its sign determines properties of the roots of (1.5). The number $|(N-p+\alpha) / p|^{p}-\mu$ plays a similar role for (1.9). Indeed, by [10, Proposition 1.3], we have the following: if $|(N-p+\alpha) / p|^{p}-\mu<0$, then (1.9) has no real root; if $|(N-p+\alpha) / p|^{p}-\mu=0$, then (1.9) has a unique real root $\lambda=(N-p+\alpha) / p$; if $|(N-p+\alpha) / p|^{p}-\mu>0$, then (1.9) has exact two real roots. 
Equation (1.9) with $\alpha=0$ appeared in the paper by Abdellaoui, Felli and Peral [2] and they studied the asymptotic behavior of radial solutions to

$$
\Delta_{p} u+\frac{\lambda}{|x|^{p}} u^{p-1}+u^{\frac{N p}{N-p}-1}=0 .
$$

See, also, Xiang [13].

The asymptotic behavior of solutions to equation

$$
\left(\phi_{p}\left(x^{\prime}\right)\right)^{\prime}+\frac{\mu}{t^{p}} \phi_{p}(x)=0,
$$

which is (1.2) with $N-1+\alpha=0$, has been investigated by Elbert 4 and Došlý and Řehák [3].

Now let $u(r)$ be a solution of (1.2). We set

$$
x(t):=u\left(e^{t}\right) .
$$

Then

$$
\left(\phi_{p}\left(x^{\prime}\right)\right)^{\prime}+(N-p+\alpha) \phi_{p}\left(x^{\prime}\right)+\mu \phi_{p}(x)=0 .
$$

Moreover we set

$$
y(t):=\phi_{p}\left(x^{\prime}(t)\right)=\phi_{p}\left(e^{t} u^{\prime}\left(e^{t}\right)\right)
$$

Then we obtain

$$
\left\{\begin{array}{l}
x^{\prime}=\phi_{p^{*}}(y), \\
y^{\prime}=-\mu \phi_{p}(x)-(N-p+\alpha) y,
\end{array}\right.
$$

where $p^{*}$ is a positive number satisfying $(1 / p)+\left(1 / p^{*}\right)=1$ and we note that $p^{*}>1$ and $\phi_{p^{*}}$ is the inverse function of $\phi_{p}$. The asymptotic behavior of solutions to the following system

$$
\left\{\begin{array}{l}
x^{\prime}=a x+b \phi_{p^{*}}(y) \\
y^{\prime}=c \phi_{p}(x)+d y
\end{array}\right.
$$

has been studied in [10, where $a, b, c, d \in \mathbf{R}$. Applying their results, we can obtain Proposition 1.1 and Theorems 1.1 1.3. which will be shown in Section 2.

In Section 3, we study the rectifiability of oscillatory solutions to

$$
\left(\phi_{p}\left(y^{\prime}\right)\right)^{\prime}+\frac{a}{x} \phi_{p}\left(u^{\prime}\right)+\frac{b}{x^{\sigma}} \phi_{p}(u)=0, \quad x>0,
$$

where $p>1, a, \sigma \in \mathbf{R}$ and $b>0$. Equation (3.1) can possess nontrivial solutions having infinitely many zeros near $x=0$. We divide the length of the curve of such a solution into finite and infinite. The case where $a=0, \sigma=p$ and $b>((p-1) / p)^{p}$ still remains an open problem. Applying Theorem 1.1 we will give its answer in Section 3.

\section{Proofs of MAin Results}

In this section we give proofs of main results. To this end, we use the following Proposition A. Theorems A, B and C obtained in [10, Proposition 1.1 and Theorems 1.1 1.3. We use the following notation:

$$
\begin{gathered}
T=a+d, \quad D=\phi_{p}(a) d-\phi_{p}(b) c, \quad \Delta=\left|\frac{a}{p^{*}}-\frac{d}{p}\right|^{p}+\phi_{p}(b) c, \\
C(\lambda)=\left\{(x, y):(a-\lambda) x+b \phi_{p^{*}}(y)=0\right\} .
\end{gathered}
$$


Proposition A. For each $\left(t_{0}, x_{0}, y_{0}\right) \in \mathbf{R}^{3}$, the initial value problem (1.15) with

$$
x\left(t_{0}\right)=x_{0}, \quad y\left(t_{0}\right)=y_{0}
$$

has a unique solution on $\mathbf{R}$.

Theorem A. Let $(x(t), y(t))$ be a solution of (1.15) with (2.1) and let $\left(x_{0}, y_{0}\right) \neq$ $(0,0)$. Assume that $\Delta<0$. Then $(x(t), y(t))$ is rotating infinitely around the origin in a clockwise [respectively counter-clockwise] direction as $t \rightarrow \infty$ when $b>0$ $[$ respectively $b<0]$, and

$$
e^{-\frac{T}{p} t} x(t) \quad \text { and } \quad e^{-\frac{T}{p^{*}} t} y(t)
$$

are periodic with period $L$ for some constant $L>0$, which depends on only $a, b, c$, $d$ and $p$.

Theorem B. Let $(x(t), y(t))$ be a solution of (1.15) with (2.1) and let $\left(x_{0}, y_{0}\right) \neq$ $(0,0)$. Assume that $\Delta=0$ and $b c \neq 0$. Then the following (i) and (ii) hold:

(i) if $\left(x_{0}, y_{0}\right) \in C(T / p)$, then

$$
(x(t), y(t))=\left(x_{0} e^{\frac{T}{p}\left(t-t_{0}\right)}, y_{0} e^{\frac{T}{p^{*}}\left(t-t_{0}\right)}\right) \in C(T / p), \quad t \in \mathbf{R} ;
$$

(ii) if $\left(x_{0}, y_{0}\right) \notin C(T / p)$, then $(x(t), y(t)) \notin C(T / p)$ for $t \in \mathbf{R}$ and

$$
\lim _{t \rightarrow \infty}\left(t^{-\frac{2}{p}} e^{-\frac{T}{p} t} x(t), t^{-\frac{2}{p^{*}}} e^{-\frac{T}{p^{*}} t} y(t)\right)=\left(x_{1}, y_{1}\right)
$$

for some $\left(x_{1}, y_{1}\right) \in C(T / p)$ with $x_{1} \neq 0$.

Theorem C. Let $(x(t), y(t))$ be a solution of (1.15) with (2.1) and let $\left(x_{0}, y_{0}\right) \neq$ $(0,0)$. Assume that $\Delta>0$ and $b c \neq 0$. Then the equation

$$
\phi_{p}(\lambda-a)[(p-1) \lambda-d]-\phi_{p}(b) c=0
$$

has exact two real roots $\lambda=\lambda_{1}, \lambda_{2}$ with $\lambda_{1}<\lambda_{2}$, and the following (i) and (ii) hold:

(i) if $\left(x_{0}, y_{0}\right) \in C\left(\lambda_{i}\right)$ for some $i \in\{1,2\}$, then

$$
(x(t), y(t))=\left(x_{0} e^{\lambda_{i}\left(t-t_{0}\right)}, y_{0} e^{\lambda_{i}(p-1)\left(t-t_{0}\right)}\right) \in C\left(\lambda_{i}\right), \quad t \in \mathbf{R} ;
$$

(ii) if $\left(x_{0}, y_{0}\right) \notin C\left(\lambda_{1}\right) \cup C\left(\lambda_{2}\right)$, then $(x(t), y(t)) \notin C\left(\lambda_{1}\right) \cup C\left(\lambda_{2}\right)$ for $t \in \mathbf{R}$ and

$$
\lim _{t \rightarrow \infty}\left(e^{-\lambda_{2} t} x(t), e^{-\lambda_{2}(p-1) t} y(t)\right)=\left(x_{1}, y_{1}\right)
$$

for some $\left(x_{1}, y_{1}\right) \in C\left(\lambda_{2}\right)$ with $x_{1} \neq 0$.

Hereafter $u(r)$ is a nontrivial solution of (1.2). Then $(x(t), y(t))=$ $\left(u\left(e^{t}\right), \phi_{p}\left(e^{t} u^{\prime}\left(e^{t}\right)\right)\right)$ is a nontrivial solution of (1.14). Applying Proposition $\mathrm{A}$. we obtain Proposition 1.1 immediately.

When $a=0, b=1, c=-\mu$ and $d=-(N-p+\alpha)$, system (1.15) becomes (1.14) and we have

$$
T=-(N-p+\alpha), \quad D=\mu, \quad \Delta=\left|\frac{N-p+\alpha}{p}\right|^{p}-\mu,
$$

and

$$
C(\lambda)=\left\{(x, y):-\lambda x+\phi_{p^{*}}(y)=0\right\} .
$$


Now we prove Theorem 1.1. Assume that $\mu>|(N-p+\alpha) / p|^{p}$. Then $\Delta<0$. Theorem $\mathrm{A}$ implies that

$$
C(t):=e^{\frac{N-p+\alpha}{p} t} x(t) \quad \text { and } \quad S(t):=\phi_{p^{*}}\left(e^{\frac{N-p+\alpha}{p^{*}} t} y(t)\right)
$$

are sign-changing periodic functions with some period $L>0$. Since $(x(t), y(t))=$ $\left(u\left(e^{t}\right), \phi_{p}\left(e^{t} u^{\prime}\left(e^{t}\right)\right)\right)$, we conclude that

$$
u(r)=r^{-\frac{N-p+\alpha}{p}} C(\log r) \text { and } u^{\prime}(r)=r^{-\frac{N-p+\alpha}{p}-1} S(\log r) .
$$

Consequently, we obtain Theorem 1.1 .

Next we give a proof of Theorem [1.2. Assume that $\mu=|(N-p+\alpha) / p|^{p}$. Then $\Delta=0$. Let $t_{0}=\log r_{0}$. From Theorem B it follows that the following (i) and (ii) hold: (i) if $((N-p+\alpha) / p) x\left(t_{0}\right)+\phi_{p^{*}}\left(y\left(t_{0}\right)\right)=0$, then

$$
x(t)=x\left(t_{0}\right) e^{-\frac{N-p+\alpha}{p}\left(t-t_{0}\right)}, \quad t \in \mathbf{R} ;
$$

(ii) if $((N-p+\alpha) / p) x\left(t_{0}\right)+\phi_{p^{*}}\left(y\left(t_{0}\right)\right) \neq 0$, then

$$
\lim _{t \rightarrow \infty}\left(t^{-\frac{2}{p}} e^{\frac{N-p+\alpha}{p} t} x(t), t^{-\frac{2}{p^{*}}} e^{\frac{N-p+\alpha}{p^{*}} t} y(t)\right)=\left(c_{1}, d_{1}\right)
$$

for some $\left(c_{1}, d_{1}\right)$ with $((N-p+\alpha) / p) c_{1}+\phi_{p^{*}}\left(d_{1}\right)=0$ and $c_{1} \neq 0$. Recalling that $(x(t), y(t))=\left(u\left(e^{t}\right), \phi_{p}\left(e^{t} u^{\prime}\left(e^{t}\right)\right)\right)$, we find that $((N-p+\alpha) / p) x\left(t_{0}\right)+\phi_{p^{*}}\left(y\left(t_{0}\right)\right)=$ 0 is equivalent to $r_{0} u^{\prime}\left(r_{0}\right)=-((N-p+\alpha) / p) u\left(r_{0}\right)$ and then we obtain (i) of Theorem 1.2. Now we suppose that $r_{0} u^{\prime}\left(r_{0}\right) \neq-((N-p+\alpha) / p) u\left(r_{0}\right)$. Then $((N-p+\alpha) / p) x\left(t_{0}\right)+\phi_{p^{*}}\left(y\left(t_{0}\right)\right) \neq 0$ and by (2.3) we obtain (1.7). Next we set $(X(s), Y(s))=(x(-s),-y(-s))$. Then $(X(s), Y(s))$ is a nontrivial solution of

$$
\left\{\begin{array}{l}
X^{\prime}=\phi_{p^{*}}(Y), \\
Y^{\prime}=-\mu \phi_{p}(X)+(N-p+\alpha) Y .
\end{array}\right.
$$

We note that system (2.4) is system (1.14) replacing $(N-p+\alpha)$ with $-(N-p+\alpha)$ and find that $(-(N-p+\alpha) / p) X\left(s_{0}\right)+\phi_{p^{*}}\left(Y\left(s_{0}\right)\right) \neq 0$ with $s_{0}=-t_{0}$. Hence we can apply Theorem B to (2.4) and then

$$
\lim _{s \rightarrow \infty}\left(s^{-\frac{2}{p}} e^{\frac{-(N-p+\alpha)}{p} s} X(s), s^{-\frac{2}{p^{*}}} e^{\frac{-(N-p+\alpha)}{p^{*}} s} Y(s)\right)=\left(c_{2}, d_{2}\right)
$$

for some $\left(c_{2}, d_{2}\right)$ with $(-(N-p+\alpha) / p) c_{2}+\phi_{p^{*}}\left(d_{2}\right)=0$ and $c_{2} \neq 0$. Since $(X(s), Y(s))=\left(u\left(e^{-s}\right),-\phi_{p}\left(e^{-s} u^{\prime}\left(e^{-s}\right)\right)\right)$, we obtain (1.8). The proof of Theorem 1.2 is complete.

Finally we suppose that $\mu<|(N-p+\alpha) / p|^{p}$. Then $\Delta>0$. Let $t_{0}=\log r_{0}$. Theorem [C implies that (1.9) has exact two real roots $\lambda=\lambda_{1}, \lambda_{2}$ with $\lambda_{1}<\lambda_{2}$, and the following (i) and (ii) hold:

(i) if $\lambda_{i} x\left(t_{0}\right)=\phi_{p^{*}}\left(y\left(t_{0}\right)\right)$ for some $i \in\{1,2\}$, then

$$
x(t)=x\left(t_{0}\right) e^{\lambda_{i}\left(t-t_{0}\right)}, \quad t \in \mathbf{R} ;
$$

(ii) if $\lambda_{i} x\left(t_{0}\right) \neq \phi_{p^{*}}\left(y\left(t_{0}\right)\right)$ for each $i \in\{1,2\}$, then

$$
\lim _{t \rightarrow \infty}\left(e^{-\lambda_{2} t} x(t), e^{-\lambda_{2}(p-1) t} y(t)\right)=\left(c_{1}, d_{1}\right)
$$

for some $\left(c_{1}, d_{1}\right)$ with $\lambda_{2} c_{1}=\phi_{p^{*}}\left(d_{1}\right)$ and $c_{1} \neq 0$. 
By $(x(t), y(t))=\left(u\left(e^{t}\right), \phi_{p}\left(e^{t} u^{\prime}\left(e^{t}\right)\right)\right)$, we see that $\lambda_{i} x\left(t_{0}\right)=\phi_{p^{*}}\left(y\left(t_{0}\right)\right)$ is equivalent to $r_{0} u^{\prime}\left(r_{0}\right)=\lambda_{i} u\left(r_{0}\right)$. Therefore, (i) of Theorem 1.3 holds. Next we assume that $r_{0} u^{\prime}\left(r_{0}\right) \neq \lambda_{i} u\left(r_{0}\right)$ for each $i \in\{1,2\}$. Then $\lambda_{i} x\left(t_{0}\right) \neq \phi_{p^{*}}\left(y\left(t_{0}\right)\right)$ and hence (2.6) holds for some $\left(c_{1}, d_{1}\right)$ with $\lambda_{2} c_{1}=\phi_{p^{*}}\left(d_{1}\right)$ and $c_{1} \neq 0$, which implies that (1.10) holds. Now we set $(X(s), Y(s))=(x(-s),-y(-s))$. Then $(X(s), Y(s))$ is a nontrivial solution of (2.4) and satisfies $\lambda_{i} X\left(s_{0}\right)+\phi_{p^{*}}\left(Y\left(s_{0}\right)\right) \neq 0$ with $s_{0}=-t_{0}$. Moreover, $\lambda=-\lambda_{2},-\lambda_{1}$ are real solutions of

$$
(p-1)|\lambda|^{p}+(-(N-p+\alpha))|\lambda|^{p-2} \lambda+\mu=0
$$

and $-\lambda_{2}<-\lambda_{1}$. Consequently, Theorem C implies that

$$
\lim _{s \rightarrow \infty}\left(e^{\lambda_{1} s} X(s), e^{\lambda_{1}(p-1) s} Y(s)\right)=\left(c_{2}, d_{2}\right)
$$

for some $\left(c_{2}, d_{2}\right)$ with $\lambda_{1} c_{2}+\phi_{p^{*}}\left(d_{2}\right)=0$ and $c_{2} \neq 0$, which means that (1.11) holds. Proposition 1.3 in [10] implies (a)-(c) in Theorem 1.3 immediately. The proof of Theorem 1.3 is complete.

\section{ReCTIFIABiLity of SOLUtions}

In this section, we consider the rectifiability of oscillatory solutions to

$$
\left(\phi_{p}\left(y^{\prime}\right)\right)^{\prime}+\frac{a}{x} \phi_{p}\left(u^{\prime}\right)+\frac{b}{x^{\sigma}} \phi_{p}(u)=0, \quad x>0,
$$

where $p>1, a, \sigma \in \mathbf{R}$ and $b>0$. A solution $y$ of (3.1) is said to be oscillatory near $x=0$ if there exists $\left\{x_{n}\right\}_{n=1}^{\infty}$ such that $y\left(x_{n}\right)=0$ for $n \in \mathbf{N}$ and $x_{n} \rightarrow 0$ as $n \rightarrow \infty$. Otherwise, it is said to be nonoscillatory near $x=0$. A solution $y$ of (3.1) is said to be rectifiable [resp. nonrectifiable] oscillatory near $x=0$ if $y$ is oscillatory near $x=0$ and the length of the graph of $y$ on $(0,1]$ is finite [resp. infinite], that is,

$$
\int_{0}^{1} \sqrt{1+\left|y^{\prime}(x)\right|^{2}} d x<\infty \quad[\text { resp. }=\infty] .
$$

On the oscillatory of solutions to equation (3.1) with $a=0$

$$
\left(\phi_{p}\left(y^{\prime}\right)\right)^{\prime}+\frac{b}{t^{\sigma}} \phi_{p}(x)=0, \quad x>0,
$$

we have the following result. (See, for example, [3].)

Theorem D. Every nontrivial solution of (3.2) is nonoscillatory near $x=0$ if one of the following (i)-(iii) holds:

(i) $\sigma>p$;

(ii) $\sigma=p$ and $b \leq((p-1) / p)^{p}$.

Every nontrivial solution of (3.2) is oscillatory near $x=0$ if one of the following (i)-(iii) holds:

(i) $\sigma<p$;

(ii) $\sigma=p$ and $b>((p-1) / p)^{p}$.

On the rectifiability of oscillatory solutions to (3.2), Pašić and Wong [12] established the following result.

Theorem E. If $p<\sigma<p^{2}$, then every nontrivial solution of (3.2) is rectifiable oscillatory near $x=0$. If $\sigma \geq p^{2}$, then every nontrivial solution of (3.2) is nonrectifiable oscillatory near $x=0$. 
For the case where $\sigma=p$ and $b>((p-1) / p)^{p}$, Theorem $\mathrm{D}$ implies that every nontrivial solution of (3.2) is oscillatory near $x=0$, but its rectifiability is open, and it is natural to expect that every nontrivial solution of (3.2) is rectifiable oscillatory near $x=0$ in view of Theorem E By the following result, we conclude that it is true.

Theorem 3.1. labelthm3.1 Assume $b>|(p-1-a) / p|^{p}$ and $\sigma=p$. Then the following (i) and (ii) hold:

(i) if a $<p-1$, then every nontrivial solution of (3.1) is rectifiable oscillatory near $x=0$;

(ii) if $a \geq p-1$, then every nontrivial solution of (3.1) is nonrectifiable oscillatory near $x=0$.

Proof. Let $y(x)$ be a nontrivial solution of (3.1). We note that equation (3.1) is equation (1.2) with $\sigma=p, \alpha=a-N+1$ and $\mu=b$. From Theorem 1.1, there exist sign-changing periodic functions $C, S$ with some period $L>0$ such that

$$
y(x)=x^{\frac{p-1-a}{p}} C(\log x), \quad y^{\prime}(x)=x^{\frac{p-1-a}{p}-1} S(\log x), \quad x>0 .
$$

Hence, $y(x)$ is oscillatory near $x=0$.

First we assume that $a<p-1$. Since $|S(t)| \leq M$ for $t \in \mathbf{R}$ for some $M>0$, using the inequality $\sqrt{1+x^{2}} \leq 1+|x|$, we conclude that

$$
\begin{aligned}
\int_{0}^{1} \sqrt{1+\left|y^{\prime}(x)\right|^{2}} d x & \leq \int_{0}^{1}\left(1+\left|y^{\prime}(x)\right|\right) d x \\
& =1+\int_{0}^{1}\left|x^{\frac{p-1-a}{p}-1} S(\log x)\right| d x \\
& \leq 1+M \int_{0}^{1} x^{\frac{p-1-a}{p}-1} d x \\
& =1+\frac{M p}{p-1-a} .
\end{aligned}
$$

Therefore, $y(x)$ is rectifiable oscillatory near $x=0$.

Now we suppose that $a \geq p-1$. Recalling $S$ is a periodic function with the period $L>0$, we find that

$$
\int_{-m L}^{-(m-1) L}|S(t)| d t=\int_{0}^{L}|S(t)| d t>0, \quad m \in \mathbf{N} .
$$

Hence, we observe that, for $n \in \mathbf{N}$,

$$
\begin{aligned}
\int_{e^{-n L}}^{1} \sqrt{1+\left|y^{\prime}(x)\right|^{2}} d x \geq \int_{e^{-n L}}^{1}\left|y^{\prime}(x)\right| d x & =\int_{e^{-n L}}^{1}\left|x^{\frac{p-1-a}{p}-1} S(\log x)\right| d x \\
& \geq \int_{e^{-n L}}^{1} x^{-1}|S(\log x)| d x \\
& =\int_{-n L}^{0}|S(t)| d t \\
& =n \int_{0}^{L}|S(t)| d t
\end{aligned}
$$


which implies that

$$
\lim _{n \rightarrow \infty} \int_{e^{-n L}}^{1} \sqrt{1+\left|y^{\prime}(x)\right|^{2}} d x=\infty .
$$

Consequently, $y(x)$ is nonrectifiable oscillatory near $x=0$.

\section{REFERENCES}

[1] B. Abdellaoui, E. Colorado, and I. Peral, Some improved Caffarelli-Kohn-Nirenberg inequalities, Calc. Var. Partial Differential Equations 23 (2005), no. 3, 327-345, DOI 10.1007/s00526004-0303-8. MR2142067

[2] Boumediene Abdellaoui, Veronica Felli, and Ireneo Peral, Existence and nonexistence results for quasilinear elliptic equations involving the p-Laplacian (English, with English and Italian summaries), Boll. Unione Mat. Ital. Sez. B Artic. Ric. Mat. (8) 9 (2006), no. 2, 445-484. MR2233146

[3] Ondřej Došlý and Pavel Řehák, Half-linear differential equations, North-Holland Mathematics Studies, vol. 202, Elsevier Science B.V., Amsterdam, 2005. MR2158903

[4] Á. Elbert, Asymptotic behaviour of autonomous half-linear differential systems on the plane, Studia Sci. Math. Hungar. 19 (1984), no. 2-4, 447-464. MR874513

[5] Zhaosheng Feng, Cheng Tan, and Lei Wei, Uniqueness and asymptotic behavior of positive solution of quasilinear elliptic equations with Hardy potential, Nonlinear Anal. 202 (2021), Paper No. 112152, 24, DOI 10.1016/j.na.2020.112152. MR.4156976

[6] Pigong Han, Quasilinear elliptic problems with critical exponents and Hardy terms, Nonlinear Anal. 61 (2005), no. 5, 735-758, DOI 10.1016/j.na.2005.01.030. MR2130063

[7] Cheng-Jun He and Chang-Lin Xiang, Asymptotic behaviors of solutions to quasilinear elliptic equations with Hardy potential, J. Math. Anal. Appl. 441 (2016), no. 1, 211-234, DOI 10.1016/j.jmaa.2016.03.082. MR.3488055

[8] Toshio Horiuchi and Peter Kumlin, On the Caffarelli-Kohn-Nirenberg-type inequalities involving critical and supercritical weights, Kyoto J. Math. 52 (2012), no. 4, 661-742, DOI 10.1215/21562261-1728839. MR2998908

[9] Susana Merchán and Luigi Montoro, Remarks on the existence of solutions to some quasilinear elliptic problems involving the Hardy-Leray potential, Ann. Mat. Pura Appl. (4) 193 (2014), no. 2, 609-632, DOI 10.1007/s10231-012-0293-7. MR3180936

[10] M. Onitsuka and S. Tanaka, Characteristic equation for autonomous planar half-linear differential systems, Acta Math. Hungar. 152 (2017), no. 2, 336-364, DOI 10.1007/s10474-0170722-6. MR 3682888

[11] Francescantonio Oliva, Berardino Sciunzi, and Giusi Vaira, Radial symmetry for a quasilinear elliptic equation with a critical Sobolev growth and Hardy potential (English, with English and French summaries), J. Math. Pures Appl. (9) 140 (2020), 89-109, DOI 10.1016/j.matpur.2020.06.004. MR4124427

[12] Mervan Pašić and James S. W. Wong, Rectifiable oscillations in second-order halflinear differential equations, Ann. Mat. Pura Appl. (4) 188 (2009), no. 3, 517-541, DOI 10.1007/s10231-008-0087-0. MR2512161

[13] Chang-Lin Xiang, Asymptotic behaviors of solutions to quasilinear elliptic equations with critical Sobolev growth and Hardy potential, J. Differential Equations 259 (2015), no. 8, 3929-3954, DOI 10.1016/j.jde.2015.05.007. MR3369267

Matsue Yamamoto Metal Co., Ltd, Hokuryo-Cho 30, Matsue-Shi, Shimane 690-0816, JAPAN

Mathematical Institute, Tohoku University, Aoba 6-3, Aramaki, Aoba-ku, Sendai 980-8578, JAPAN

Email address: satoshi.tanaka.d4@tohoku.ac.jp 